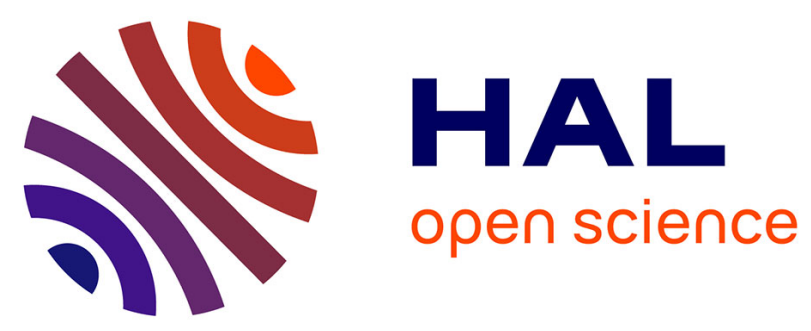

\title{
Scalable versus Accurate Physical Layer Modeling in Wireless Network Simulations
}

\author{
Elyes Ben Hamida, Guillaume Chelius, Jean-Marie Gorce
}

\section{To cite this version:}

Elyes Ben Hamida, Guillaume Chelius, Jean-Marie Gorce. Scalable versus Accurate Physical Layer Modeling in Wireless Network Simulations. 22nd ACM/IEEE/SCS workshop on Principles of Advanced and Distributed Simulation (PADS 2008), ACM/IEEE/SCS, Jun 2008, Roma, Italy. pp.127134, 10.1109/PADS.2008.12 . inria-00384134

\section{HAL Id: inria-00384134 https://hal.inria.fr/inria-00384134}

Submitted on 14 May 2009

HAL is a multi-disciplinary open access archive for the deposit and dissemination of scientific research documents, whether they are published or not. The documents may come from teaching and research institutions in France or abroad, or from public or private research centers.
L'archive ouverte pluridisciplinaire HAL, est destinée au dépôt et à la diffusion de documents scientifiques de niveau recherche, publiés ou non, émanant des établissements d'enseignement et de recherche français ou étrangers, des laboratoires publics ou privés. 


\title{
Scalable versus Accurate Physical Layer Modeling in Wireless Network Simulations
}

\author{
Elyes Ben Hamida, Guillaume Chelius and Jean-Marie Gorce \\ ARES INRIA / CITI, INSA Lyon, F-69621, France \\ \{elyes.ben-hamida, jean-marie.gorce\}@insa-lyon.fr, guillaume.chelius@inria.fr
}

\begin{abstract}
In wireless networking, due to the high complexity of analytical and theoretical models, simulations are generally considered as the most convenient methodology for performance evaluation. Nonetheless, the physical complexity of the wireless medium induces a clear tradeoff between accuracy and scalability in a wireless network simulator design. In this paper, we focus on this tradeoff and study the impact of physical layer (PHY) modeling accuracy in the computational cost of simulations. We first discuss the main aspects of the wireless medium and briefly show how they have been handled in existing simulators. Then, we introduce a flexible and modular PHY simulation framework to analyze in more details their influence on the scalability of simulations.
\end{abstract}

\section{Introduction}

In wireless multi-hop networking, due to the highcomplexity of analytical and theoretical models, simulations are generally considered as the most convenient methodology to explore the behavior of protocols and distributed applications. Nonetheless, the complexity of the physical phenomena constituting the radio medium introduces a tradeoff between accuracy and computational cost in wireless network simulation. Several wireless network simulators have been proposed in the last years. Examples are NS-2, GloMoSim [3], JiST/SWANS [4], GTSNetS [12], etc. They all provide an advanced and complete simulation environment to investigate and evaluate networking protocols and wireless systems. However, the complexity of the wireless physical layer (PHY) leads to implementation choices during the simulators design. As a consequence, the PHY simulation accuracy varies drastically from one simulator to another.

As it has been highlighted in previous publications $[6,10$, 15], these variations largely impact on the results of a simulation. A correct modeling of the PHY layer is then crucial for confidence in the simulation results. Nonetheless, most of wireless network simulators are still based on inaccurate PHY models. The reason that generally prevails to justify this low-accuracy is scalability.

In this paper, we study the impact of PHY modeling accuracy on the computational cost of simulations. The question we raise is what is the real cost of PHY simulation accuracy? We deliberately keep aside optimizations and scalability of the node and protocol aspects of simulations which have been the subject of other studies [8]. To evaluate the PHY tradeoff, we introduce a flexible and modular PHY simulation framework, called WSNet ${ }^{1}$. We chose not to analyze the PHY tradeoff through the study of an existing simulator as none of them offer a sufficient diversity in PHY models. Comparing several existing simulators would not have helped, as they differ in many other aspects than PHY modeling.

The remainder of this paper is organized as follows. In section 2, we discuss the main aspects of wireless communications. Then, in Section 3, we review some common wireless network simulators and briefly compare their PHY modeling. Next, the WSNet simulation framework is presented in Section 4. In Section 5 we investigate the impact of the PHY modeling on the simulation speedup. Finally, the tradeoff involving accuracy and scalability is illustrated in Section 6.

\section{Toward a precise physical layer modeling}

For the sake of realism and confidence in simulation results, an accurate PHY modeling is a key point. In analytical studies as in simulations, the disk model has long prevailed. It relies on a set of strong assumptions: time stationarity $l_{i j}(t)=l_{i j}$, independence $l_{i j}=f\left(x_{i}, x_{j}\right)$, switched link (on/off) $l_{i j} \in\{0,1\}$, symmetry $l_{i j}=l_{j i}$, isotropy $l_{i j}=f\left(x_{i}, d_{i j}\right)$ and homogeneity $l_{i j}=f\left(d_{i j}\right)$, where $l_{i j}$ refers to the radio link between nodes $i$ and $j$ and $d_{i j}$ to the geometric distance between $i$ and $j$.

\footnotetext{
${ }^{1}$ WSNet is available at: http://wsnet.gforge.inria.fr.
} 
The disk model provides the radio network with three axioms: the radio range is constant, the radio link is switched, and the network is interference free. If the asset of this model holds in its simplicity for both theoretical studies and simulations, the resulting simulations are far from realistic. Nonetheless, improving this model is not a trivial task as a hard tradeoff between complexity and realism holds. Basically, this model can be improved by relaxing either of the three previously stated axioms, as discussed below.

\subsection{Radio range modeling}

The range of a radio system is based upon the definition of a signal to noise ratio (SNR) threshold noted $\bar{\gamma}_{l i m}$. If the system is interference free, the range is a constant and the radio link is defined by:

$$
\begin{aligned}
& l_{i j}: \Omega^{2} \mapsto B=\{0,1\} \\
& \left(x_{i}, x_{j}\right) \mapsto l\left(x_{i}, x_{j}\right)= \begin{cases}1 & \text { if } \bar{\gamma}_{i j} \geq \bar{\gamma}_{l i m} \\
0 & \text { else }\end{cases}
\end{aligned}
$$

where the SNR $\bar{\gamma}_{i j}$ is given by: $\bar{\gamma}_{i j}=h_{i j} \cdot \frac{P_{i}}{N_{j}}$, where $h_{i j}$ is the path-loss and $P_{i}$ and $N_{j}$ are the transmission power and the noise level respectively.

The transceiver properties. They are the transmission power $P_{i}$, the noise level $N_{i}$, the antenna gain and its radiation pattern $g_{i}(\theta, \phi)$. Variations of $P_{i}, N_{i}$ or antenna gains affect the spatial homogeneity assumption (and so far the symmetry), which means that all nodes not further have the same range. Note that a non-uniform noise level $N_{j}$ is highly probable for low cost small radio systems. The isotropy which is not statistically affected by these parameters does not further hold if the radiation patterns of the antennas are introduced according to: $h_{i j}=$ $g\left(x_{i}, x_{j}\right) \cdot g_{i}\left(\theta_{i j}, \phi_{i j}\right) \cdot g_{j}\left(\theta_{j i}, \phi_{j i}\right)$, where $g\left(x_{i}, x_{j}\right)$ is the propagation path-loss. It should be noted that $3 \mathrm{D}$ radiation pattern and 3D distribution of radio systems is pertinent for the simulation of small indoor environments for example.

Propagation models. The simplest model refers to the line of sight (LOS) scenario but in urban and indoor environments, more complicated scenarios occur due to shadowing and multiple paths. Two complementary approaches can be used to deal with propagation.

The former approach relies on a deterministic modeling of the wave propagation and provides fine simulations of any environment. The most usual algorithms are ray-tracing based [1] but discrete methods have been also proposed [9]. The high accuracy of these methods is definitely balanced by their high computational cost. Another limitation of purely deterministic models is that simulating one real environment is often too specific.Thus, the later approach relies on a statistical description complementing the deterministic model. A stochastic variable $\mathbf{s}_{i j}$ is then introduced in the propagation path-loss to handle shadowing: $\mathbf{g}_{i j}=g_{i j} \cdot \mathbf{s}_{i j}$. The most usual model is the log-normal shadowing. A very challenging issue with shadowing is to introduce a spatial correlation between radio links which is not found in current simulators. Thus, $\mathbf{s}_{i j}$ should be a spatial correlated stochastic process constant in time; it is not the case in some works as shadowing is often confused with fading. Fading refers to SNR time variations due to multi-path interference. It has a leading role in wireless communications and is introduced also as a stochastic variable $\mathbf{f}_{i j}: \mathbf{g}_{i j}(t)=g_{i j} \cdot \mathbf{s}_{i j} \cdot \mathbf{f}_{i j}(t)$. This late variable is not spatially correlated as it relies on small scale phenomenon. Meanwhile, it is a time variant parameter. Considering its temporal correlation may be highly relevant [13] and represents another challenge for wireless simulators.

\subsection{Radio link modeling}

A frame error rate (FER) as a function of the mean SNR can substitutes for the SNR threshold of Equation 1. It derives from the bit error rate (BER) function which itself relies on the radio interface properties. $l_{i j}$ then relates to the probability of a successful transmission. Theoretical asymptotic expressions are well-known for various modulation techniques [16].

\subsection{Interference modeling}

Interference disturbs the packet reception at the physical layer. It appears as a crucial point in PHY simulations as final results can be strongly influenced by the interference model $[6,10,15]$. As we will see in Section 3, interference management is probably the point where current simulators differ the most largely. Sources of interference include nodes operating in the same frequency band or in different frequencies. The first type of interference is known as co-channel interference, while the latter is termed adjacent channel interference.

The most efficient approach for introducing interference consists in replacing the SNR by a signal to interference plus noise ratio, SINR, which can be derived according to:

$$
\bar{\gamma}_{i j}=h_{i j} \cdot \frac{P_{i}}{N_{j}+\sum_{k \neq i, j} h_{k j} \cdot P_{k}}
$$

The proper derivation of the SINR requires the knowledge, at a given time, of all the signals which are concurrently received at a given receiver.

To be exhaustive, it should be noted that non linear receivers (with multi-user detection for instance) [2] can outperform classical receivers in the presence of interference. In this case, it is necessary to compute the FER not from the general SINR, but rather from the vector of received powers at each node. 


\subsection{MIMO systems}

Multiple input multiple output (MIMO) interfaces are very promising and their simulation can be assessed in two ways. The former but simplest approach is referred to as the node-based approach and exploits the single antenna framework described above by adjusting the FER function according to a MIMO specific model. This approach is however not relevant for realistic systems where the radiation patterns of the multiple antennas differ from each other as well as for correlated channels. The later, referred to as the antenna-based approach, is more powerful and simulates the channel state for each antenna-to-antenna link, separately. For instance, in a $2 \times 2 \mathrm{MIMO}$ system, four channels are simulated. It is very important to take care about the correlation between these channels to have accurate simulations. In this context, modeling spatially correlated shadowing and time correlated fading is of great importance.

\section{State of the art}

Numerous wireless network simulators have been developed and are concurrently used in the wireless network research domain. In this section, the PHY modeling of four widely used simulators is presented and briefly investigated. The simulators are NS-2, GloMoSim [3], JiST/SWANS [4] and GTSNetS [14]. This review is far from being exhaustive and has been deliberately restricted for space restriction reasons. Other available simulators with wireless support are OPNET, OMNeT++, etc.

Let us consider the PHY modeling as implemented in these simulators. Due to space limitations, we restrict our focus to one of the most crucial aspects for cross-layer and protocol studies, namely interference modeling.

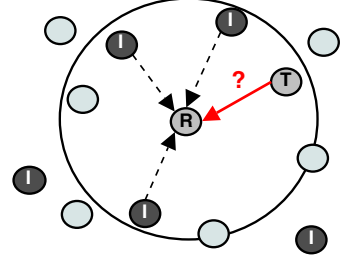

(a) Limited interference

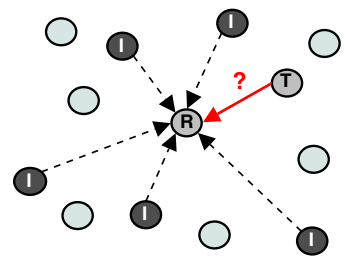

(b) Full interference
Figure 1. Limited versus full interference model (T: transmitter, $R$ : receiver and $I$ : interfering nodes).

The first step toward interference evaluation is to identify which signals are interfering with each other in order to assess the terms in the denominator of eq. 2, on the basis of timing considerations only. This set of interfering signals can be very large for large scale simulations. As a consequence, various simulators rather limit the range at which any signal can propagate and thus can interfere. In other words, disregarding the radio range model effectively used for the received signal strength computation, the simulator does not generate receptions at nodes further than a given range from the source. Consequently, the considered source cannot induce interference at nodes further than this range. This optimization, called Limited interference model as opposed to the Full interference model, is depicted in Figure 1. It privileges scalability at the cost of accuracy. This optimization is implemented in JiST/SWANS, GloMoSim, etc.

Regarding the SINR computation, several strategies have been investigated and implemented in existing simulators. They are all variations of eq. 2 regarding timing granularity. They induce a varying level of realism, precision but also complexity. They are summed up in Figure 2:

- Fig 2(a): only one SINR value is computed for the whole packet. The noise value considered for the SINR computation is the cumulative power of the signals that interfere with the considered signal at reception time. This method is the one implemented in JiST/SWANS.

- Fig 2(b): only one SINR value is computed for the whole packet. The noise value considered for the SINR computation is the cumulative power of all interfering signals weighted by the interference duration.

- Fig 2(c): only one SINR value is computed for the whole packet. The noise value considered for the SINR computation is the power of the strongest interfering signal. This method is implemented in NS-2 and is close to the collision model of GTSNetS.

- Fig 2(d): several SINR values are computed for the packet; whenever the set of interfering signals changes, a new SINR value is computed. The noise value considered for each SINR computation is the cumulative power of all concurrent interfering signals. This method is implemented in GloMoSim.

It is obvious that these interference models offer different levels of complexity, accuracy or realism. In order to better evaluate the computational cost of interference modeling, we introduce the WSNet PHY simulation framework.

Note that none of these simulators supports multichannel systems. For this purpose, the interference model in (2) should be replaced by:

$$
\bar{\gamma}_{i j}=h_{i j} \cdot \frac{P_{i}}{N_{j}+\sum_{k \neq i, j} \alpha_{i k} \cdot h_{k j} \cdot P_{k}},
$$

where $\alpha_{i k}$ stands for the rejection factor between the channels associated with signals $i$ and $k$. 


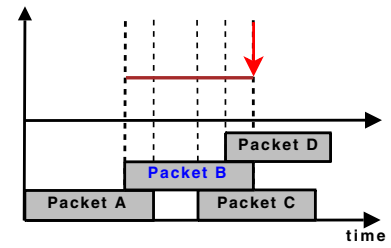

(a) $\frac{P_{B}}{P_{D}+P_{C}}$

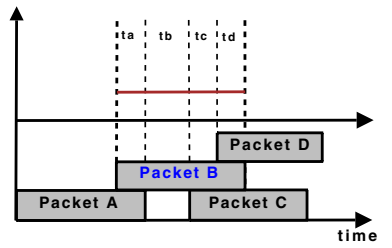

(b)

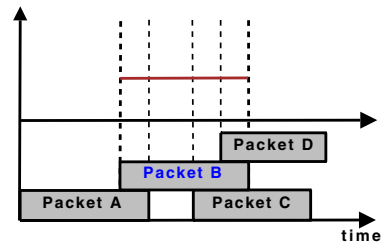

(c) $\frac{P_{B}}{\operatorname{MAX}\left(P_{A}, P_{D}, P_{C}\right)}$

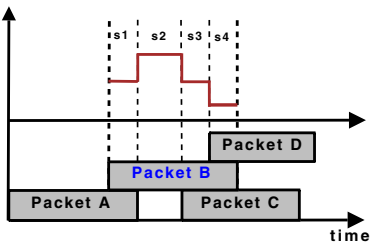

(d) $\left[\frac{P_{B}}{P_{A}},+\infty, \frac{P_{B}}{P_{C}}, \frac{P_{B}}{P_{D}+P_{C}}\right]$

Figure 2. SINR computation strategies at the node receiving the packet $B$.

\section{The WSNet PHY simulation framework}

The WSNet PHY simulation framework is well-designed for the purpose of evaluating the computational cost of the PHY modeling accuracy. The design of WSNet has been mainly guided by two constraints: modularity and flexibility. Thanks to modularity, WSNet can offer a wide range of PHY models, from ideal ones to pseudo-realistic ones.

WSNet operates either as a complete event-driven wireless network simulator, including the simulation of both the wireless nodes and the radio medium, or as a physical layer simulator with nodes being simulated by external simulators in a distributed simulation framework [7].

\subsection{PHY layer simulation}

In WSNet, a radio signal is characterized by the following values: an emission power, a symbol rate, a modulation scheme and a channel number. The PHY layer is abstracted as a combination of independent blocs which represent radio medium properties or hardware components. The radio simulation is built upon the following blocs: (i) a propagation bloc dealing with propagation aspects including pathloss, shadowing and fading according to the definitions provided in section 2; (ii) an interference bloc which implements eq. 3; (iii) modulation blocs which provide SINR-toBER conversions; and (iv) antenna blocs which implement antenna properties: loss, gain, radiation pattern, orientation and position. Note that WSNet offers a 3D spatial representation.

These blocs are black-boxes with well-defined interfaces. At simulation runtime, they are linked to dynamic libraries which effectively implement the interface. As an example, for the propagation bloc, a dynamic library implements the free space propagation model while another one provides a Rayleigh channel and a third one inputs pathloss values from an external file or an external propagation tool. More generally, this architecture is an opportunity to offer a wide range of radio medium models, from a basic ideal physical layer with no interference and no path-loss to a more realistic one including a Rayleigh channel, multiple frequencies, complex modulation schemes and smart antennas. It is thus a good opportunity to study the computational cost of using pseudo-realistic radio models in term of simulation complexity.

\subsection{Radio range simulation}

When considering a transmission initiated by an antenna $i$, WSNet generates two events at each receiving antenna. The first one notifies the apparition of the signal while the second one notifies its end. Given a receiving antenna $j$, the two events are scheduled according to the distance between the emitting and receiving antennas, the radio propagation speed and the signal symbol rate. By default, the set of receiving antennas is extended to all antennas of the network. Thus, by default, WSNet operates in a full interference mode. However, the simulation configuration may indicate a limited interference mode together with a maximum range after which receptions and interference are not propagated. This range is independent from the radio range model implemented in the propagation bloc.

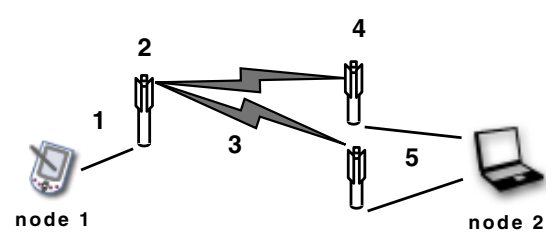

Figure 3. Signal reception

For each reception, i.e. each receiving antenna $j$, the received signal strength is computed according to the operations and properties depicted in Figure 3: (1) the emitting antenna noise $N_{i}$, (2) the emitting antenna gain $g_{i}\left(\theta_{i j}, \phi_{i j}\right)$, (3) the signal attenuation including shadowing and fading effects $\mathbf{g}_{i j}(t)$, (4) the receiving antenna gain $g_{j}\left(\theta_{j i}, \phi_{j i}\right)$ and (5) the receiving antenna noise $N_{j}$.

\subsection{Interference simulation}

Upon signal reception, after the received signal strength computation, interference is considered: SINR values are 
evaluated according to eq. 3 . WSNet divides the signal into slots and computes a SINR value for each of the slots. In order to trade accuracy for scalability and vice-versa, the number of slots can be tuned from 0 - no interference - up to the packet length - one SINR value for each signal byte. In each slot, WSNet computes a SINR according to the weighted cumulative interference model described in Figure 2(b). As a consequence, given the configured number of slots, WSNet accuracy ranges from Fig. 2(b) to Fig. 2(d).

Channels have been introduced in WSNet to support multi-channel and MIMO systems. Channels divide the radio resource in sub-resources which mutually interact. As an example, channels can be used to model frequencies in a FDMA (Frequency Division Multiple Access) network as well as codes in a CDMA (Code Division Multiple Access) one. We do not explicit what channels are but we rather explicit the correlation, i.e. how they interfere with each others. While computing the interference on channel $i$, the contribution of a signal emitted on channel $j$ is weighted by a correlation factor $\alpha_{j i}$ as given in eq. 3. $\alpha_{j i}$ values are provided by the interference bloc.

\subsection{Radio link simulation}

Upon signal reception and after each slot SINR computation, the impact of interference on the radio signal is evaluated by computing the BER values. The SINR to BER conversion is provided by the modulation bloc that characterizes the radio signal. As for the SINR, a BER value is associated with each slot of the radio signal. Based on the BER values and the slot lengths, a FER is finally computed. Contrarily to GloMoSim, WSNet forwards the radio signal to the receiving antenna whatever the FER value and whenever the first error arises. Indeed, in the majority of cases, errors are not detected until the signal is completely received or lost.

An optional feature of WSNet is to randomly introduce errors in the radio signal with a probability equal to the computed BER. In this case, an erroneous signal is transmitted to the receiving antenna. Given this feature, it becomes possible to study precisely the performance of channel coding and error correction algorithms through their implementation in the node simulation part. More generally, CDMA schemes can be either simulated statistically using channels and correlation factors or studied precisely through their implementation and the error introduction feature.

\section{Impact of the physical layer modeling}

We now study the cost of PHY modeling accuracy using WSNet, focusing on the three aspects that have been developed in Section 2: radio range modeling, interference modeling and radio link modeling. As an estimation of the com- putational cost, we use the speedup metric. The speedup of a simulation is the ratio between the logical simulated time and the effective simulation time. Unless specified otherwise, the simulations consist in 100 nodes deployed randomly in a $100 \times 100 \times 0$ area. Each node emits a $100 \mathrm{~B} / \mathrm{s}$ Constant Bit Rate (CBR) broadcast traffic through an IEEE 802.15.4 868Mhz compliant radio. No MAC protocol is executed in order to focus on the PHY simulation. The presented speedups are averaged over 100 simulations with the confidence intervals being omitted as they have been found to be negligible.

\subsection{Radio range models}

To assess the impact of radio range models on the speedup of simulations, we compare four common propagation models: a disk model (no path-loss) with transmission ranges of $60 \mathrm{~m}$ and $200 \mathrm{~m}$, the free-space model, the two-ray ground model and a Rayleigh channel with a pathloss exponent of 2 for the last three cases. Interference and modulation support are disabled. The results are drawn on Figure 4.

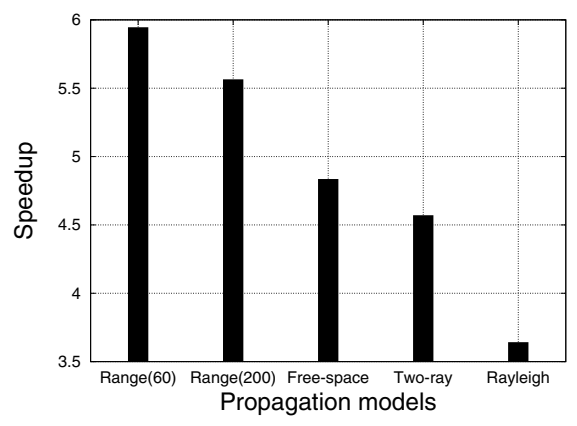

Figure 4. Impact of propagation models.

Quite obviously, a better accuracy in the radio range model induces a decrease in the simulation speedup. There are two reasons for this overhead. The first one is the computational cost associated with the radio range model. It takes more time to compute a complex path-loss with random variables than a single distance. The second one is an increase in the number of receptions generated by a single emission. Consider for example the Range(60) and Range(200) models. They have the same computational cost but the number of receivers is larger with a range of 200 meters. In a realistic modeling, this overhead is even higher as the signal reaches all nodes. If the first overhead can hardly be reduced, the second one can be reduced using the limited interference model. 


\subsection{Interference models}

Keeping the Friis propagation model, we now analyze the impact of interference simulation on the scalability of simulations. In a first step, we make the number of SINR slots vary from 0 , no interference simulation, to $n$ - one SINR per byte. Results are depicted on Figure 5. In a second step, we evaluate the cost of multi-channel support and adjacent channel interference computation. We vary the number of simultaneously simulated channels from 1 to 16 . Results are depicted on Figure 6.

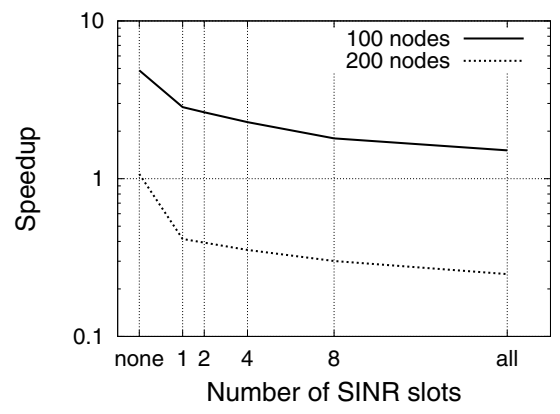

Figure 5. Impact of co-channel interference.

Support of a cumulative interference model induces a strong overhead as the speedup is roughly reduced by $50 \%$ from no interference simulation to a single SINR computation. However, in interference modeling, it seems that the first step is the one that costs the most. Indeed, from a single SINR to a per-byte computation, the extra overhead remains quasi constant for each refinement: from $4 \%$ for two SINR values to $45 \%$ for $n$ values. In consequence, once the interference price has been paid, there is no strong reason to decline paying for a better accuracy. This same conclusion holds for multi-channel support as its cost is negligible compared to the one associated with an accurate interference modeling.

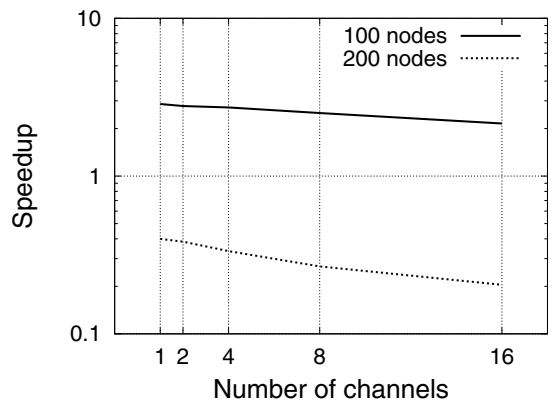

Figure 6. Impact of multi-channel interference.

\subsection{Radio link models}

In addition to interference, we now evaluate the cost of an accurate radio link modeling by integrating three modulation models in the simulations: SINR threshold, bpsk and oqpsk. The results are shown in Figure 7.

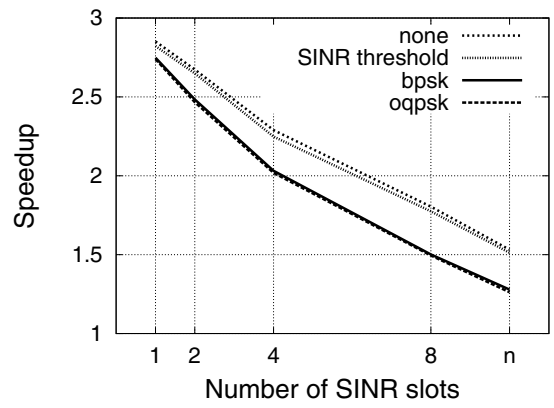

Figure 7. Impact of modulation on speedup.

As a BER value is derived from each computed SINR, the overhead induced by the radio link model is function of the interference modeling accuracy. As in Section 5.1, a realistic model induces a higher overhead: the computational cost of the erf $c$ function used in the bpsk model is much higher than a simple comparison between a SINR and a threshold. However, from a SINR threshold to a bpsk function, the speedup only decreases by 4 to $17 \%$ depending of the number of SINR slots. This price may clearly be worth the gain.

\subsection{Limited interference model}

We finally study the speedup gain that can be achieved through the use of a limited interference model. If this model decreases the accuracy of the PHY layer simulation, it is supposed to increase the scalability of simulators, justifying its use in GloMoSim, JiST/SWANS, etc. For this evaluation, we consider a network of 2000 nodes randomly deployed in a network of size $1000 \mathrm{~m} \times 1000 \mathrm{~m} \times 0 \mathrm{~m}$. No interference nor modulation is simulated. We compare the speedup achieved using the limited interference model for various maximum interference/reception ranges to the one achieved using the full interference model. Results are presented in Figure 8. In the same Figure, we also highlight the interest of using an optimized structure to maintain the node positions and to search for nodes in reception/interference range. Indeed, the complexity of range searching using a list is $O(n)$ and thus does not scale well in the number of nodes. In consequence, several optimized data structures have been proposed for the problem of range searching [5]. Two structures have been implemented in WSNet and are compared here: a db-tree (dynamic balanced tree) and a grid. A more 
complete study of optimized data structures for the range search problem can be found in [5].

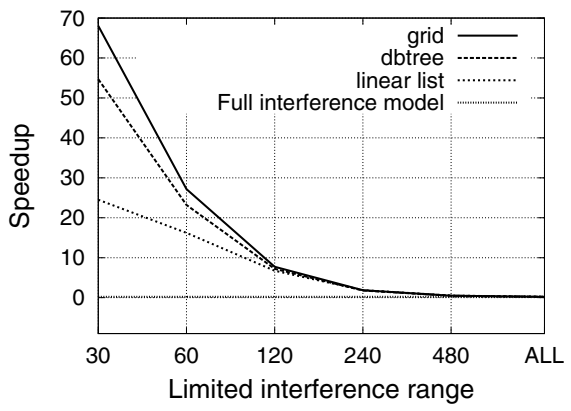

Figure 8. Space partitioning methods.

As it was already pointed out in Section 5.1, a reduction in the number of receivers induces a large gain in the simulation speedup. For a limited interference range of 30 meters, with the correct data structure, the speedup can be increased by a factor of 332 . In large-scale networks, this gain remains high even for larger ranges. With a limited range of 120 meters, a gain of 37 is still achieved. It is quite obvious that the limited interference model is a must to ensure a high scalability. However, its use raises the issue of determining a correct range (i.e., the distance limit for the propagation). As a solution, [11] proposes an empirical method to derive a range with a limited impact on the simulation accuracy.

\section{Scalability versus accuracy}

Finally, we propose a short case study to clearly summary the impact of PHY modeling on the scalability and accuracy of simulations. We consider a varying number of nodes randomly deployed in a $200 \times 200 \times 0$ area. Each node emits periodically a hello packet $(100 \mathrm{~B} / \mathrm{s})$ through an IEEE 802.15.4 868Mhz compliant radio. We consider three metrics: the speedup, the average number of discovered neighbors and the corresponding average number of connex components. The first metric allows to assess the impact of radio models on the scalability while the two latter metrics show the impact of the PHY layer modeling on the evaluation of higher level protocols in terms of network connectivity. We performed the same set of simulations with various PHY models. Starting from (i) a basic disk model (range $=50$ ) with no interference nor modulation, we slightly increased the PHY simulation accuracy through the introduction of (ii) path-loss and (iii) log-normal shadowing with a transmission power of $0 \mathrm{dBm}$. Then, we considered cumulative interference with a limited interference model (range of 50) and BPSK modulation for (iv) one slot per packet and $(v) n$ slots per packet. We finished with the $(v i)$ full interference model. Simulation results are shown on Figure 9.
As pointed out in Section 5.1, we first notice that the addition of path-loss and fading to the range model induces a low impact on the speedup. Still considering the speedup, the main gap occurs with the introduction of interference and modulation support.
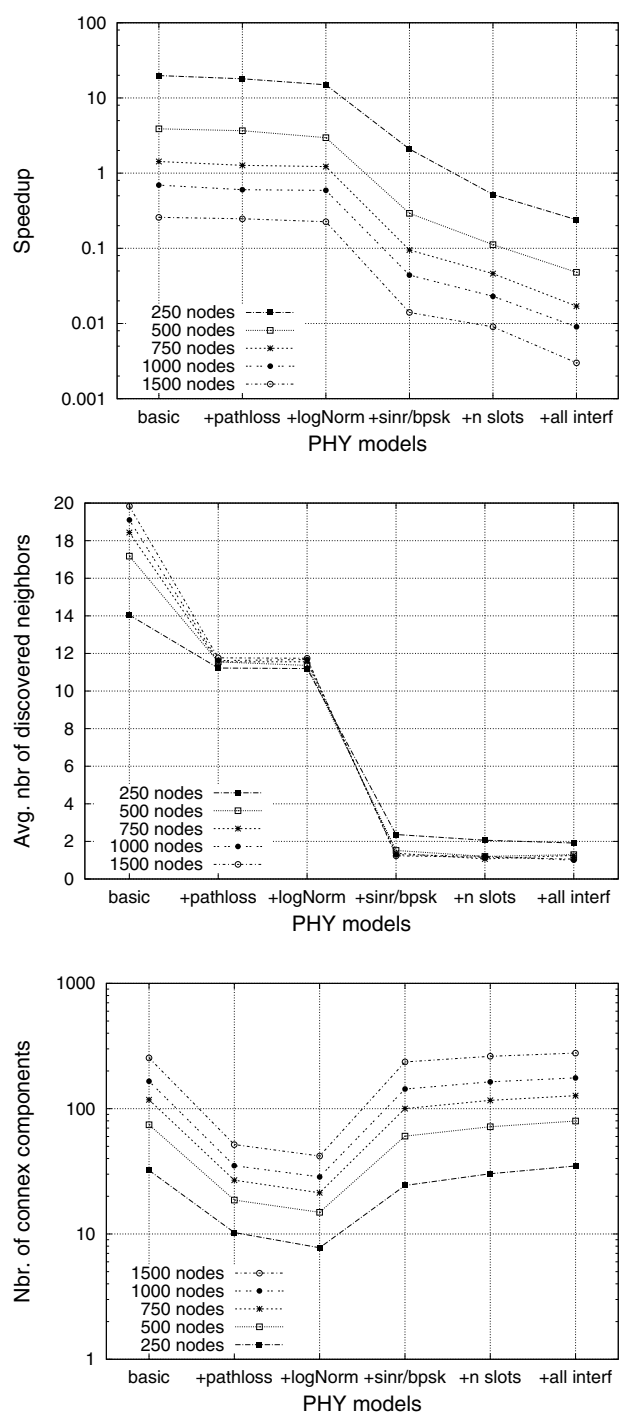

Figure 9. Impact of the PHY modeling accuracy on simulation speedup and results.

Next, as previously observed in Section 5.2, considering $n$ SINR slots instead of 1 induces a regular extra overhead. Finally, the full interference model does not induce too much overhead as the network area is small in this case. Regarding the behavior of higher level protocols under variable PHY models, it can be observed that the average number of discovered neighbors varies systematically. If the largest gap also occurs when interference and modulation 
are introduced, the results still degrade when the accuracy of the interference modeling is increased. This degradation is more important with a high number of nodes. As the number of nodes increases, so does the level of interference and its impact on higher protocols. Sensibility to PHY accuracy increases with the network size.

Considering the average number of connex components, we observe that the logical network connectivity varies with the PHY modeling. As expected, the use of a path-loss or a log-normal shadowing with no interference increases the network connectivity (i.e, low number of connex components). However, this connectivity decreases significantly (i.e, high number of connex components) when increasing the interference model accuracy, from 1 to $n$ slots, from a limited to a full model with modulation support. This underlines the importance of using accurate radio medium models when evaluating protocols, especially in large-scale networks.

\section{Conclusion}

In this paper, through the introduction of the WSNet PHY simulation framework, we have studied the impact of PHY modeling on the scalability and accuracy of wireless network simulations. To the question raised in the introduction: what is the real cost of PHY simulation accuracy ?, we can give the following conclusions. Accuracy has a variable cost depending on the considered PHY aspect. On one hand, for radio range modeling, the cost remains low, especially compared to the gain that can be achieved using realistic models. On the other hand, interference simulation induces a real high overhead. However, the extra cost remains regular when the simulation accuracy gets refined. Given the overhead of interference modeling, the cost added by accurate radio link models remains low. So as the support of multiple channels in the simulation. As a consequence, it is prejudicial to simulate interference but no realistic modulation schemes.

Given these trends, it remains up to the user choice to trade PHY accuracy for a desired scalability. However, we must keep in mind that, as highlighted in this work, the validity of simulation results highly depends on the choices made for the PHY modeling. In particular, if the use of optimizations as a limited interference model drastically increases the scalability of simulators, it does not solve the accuracy versus scalability tradeoff. The optimization performance and correctness now depends on the chosen limited range. In the future, we plan to investigate the limited interference model in more details. In parallel to the empirical model proposed by [11], we think that a stochastic theoretical analysis can help in determining an adequate range. The goal here is still to enhance the scalability of simulators without affecting the confidence in the simulation results.

\section{References}

[1] P. Almers and Al. Survey of channel and radio propagation models for wirelessmimo systems. EURASIP Journal onWireless Communications and Networking, 1:1-19, 2007.

[2] J. G. Andrews. Interference cancellation for cellular systems: a contemporary overview. IEEE Wireless Communications, 12(2):19-29, april 2005.

[3] L. Bajaj, M. Takai, R. Ahuja, and R. Bagrodia. Simulation of large-scale heterogeneous communication systems. In Proceedings of MILCOM'99, November 1999.

[4] R. Barr, Z. J. Haas, and R. van Renesse. Scalable Wireless Ad hoc Network Simulation, chapter Ch. 19, pages pp. 297311. Handbook on Theoretical and Algorithmic Aspects of Sensor, Ad hoc Wireless, and Peer-to-Peer Networks, 2005.

[5] J. L. Bentley and J. H. Friedman. Data structures for range searching. ACM Comput. Surv., 11(4):397-409, 1979.

[6] D. Cavin, Y. Sasson, and A. Schiper. On the accuracy of manet simulators. In International Workshop on Principles of Mobile Computing (POMC), Toulouse, France, Oct. 2002. ACM.

[7] G. Chelius, A. Fraboulet, and E. Fleury. Worldsens: development and prototyping tools for application specific wireless sensors networks. In International Conference on Information Processing in Sensor Networks (IPSN), Boston, USA, April 2007.

[8] Egea-Lopez and Al. Simulation scalability issues in wireless sensor networks. Communications Magazine, IEEE, 44(7):764- 73, July 2006.

[9] J.-M. Gorce, K. Jaffres-Runser, and G. de la Roche. A deterministic approach for fast simulations of indoor radio wave propagation. IEEE Transactions on Antennas and Propagation, 55(3,2):938-948, 2007.

[10] J. Heidemann, N. Bulusu, and J. Elson. Effects of detail in wireless network simulation. In Multiconference on Distributed Simulation. SCS, Jan. 2001.

[11] Z. Ji, J. Zhou, M. Takai, and R. Bagrodia. Improving scalability of wireless network simulation with bounded inaccuracies. ACM Trans. Model. Comput. Simul., 16(4):329-356, 2006.

[12] E. Ould-Ahmed-Vall, G. F. Riley, B. S. Heck, and D. Reddy. Simulation of large-scale sensor networks using gtsnets. In the 13th IEEE International Symposium on Modeling, Analysis, and Simulation of Computer and Telecommunication Systems (MASCOTS'05), 2005.

[13] P. P. Pham, S. Perreau, and A. Jayasuriya. New cross-layer design approach to ad hoc networks under Rayleigh fading. IEEE Journal on selected Areas in Communications, 23(1):28-39, 2005.

[14] G. F. Riley. Large-scale network simulations with gtnets. In Proceedings of the 2003 Winter Simulation Conference, volume 1, pages 676-684, 2003.

[15] M. Takai, J. Martin, and R. Bagrodia. Effects of wireless physical layer modeling in mobile ad hoc networks. In International Symposium on Mobile Ad Hoc Networking and Computing (MobiHoc), Long Beach, USA, Oct. 2001.

[16] Z. Wang and G. Giannakis. A simple and general parametrization quantifying performance in fading channels. IEEE trans on Communications, 51(8):1389-1398, August 2003. 